\title{
Prevalence and Determinants of Maternal Malnutrition in Africa: A Systematic Review
}

\author{
Prince L. Bestman (PLB) $)^{1,2,3}$, Atipatsa C. Kaminga (ACK) ${ }^{4,5}$, Jiayou Luo (JYL) ${ }^{1, *}$ \\ ${ }^{1}$ Department of Maternal and Child Health, Xiangya School of Public Health, Central South University, \\ Changsha, Hunan Province, P. R. of China \\ ${ }^{2}$ Phebe Hospital and School of Nursing, Bong County, Liberia, West Africa \\ ${ }^{3}$ Nurse-Anesthetist John F. Kennedy Memorial hospital, Monrovia, Liberia, West Arica \\ ${ }^{4}$ Mzuzu University, Department of Mathematics and Statistics, Private Bag 201, Luwinga, Mzuzu, Malawi \\ ${ }^{5}$ Department of Epidemiology and Health Statistics, Changsha city, Hunan province, P.R. of China
}

*Corresponding Author: Prof. Jiayou Luo, Department of Maternal and Child Health, Xiangya School of Public Health, Central South University, 110 Xiangya Rd, Changsha, Hunan, P.R. of China, Email: iiavouluo@126.com

\begin{abstract}
Introduction: Malnutrition is a serious public health condition affecting a huge proportion of reproductive age women worldwide. Prevalence of malnutrition among reproductive age females is unsatisfactorily high in most African setting.
\end{abstract}

Objective: This review was conducted to objectively investigate the prevalence and determinants of malnutrition in Africa

.Search Strategy: The advance search option was used with the keywords; prevalence, maternal malnutrition and Africa.

Selection Criteria: Cross-sectional studies Published from January 1st, 2000 to December 31st 2019 on maternal malnutrition in Africa where body mass index or Mid Upper Arm Circumference was used to predict nutritional status of study subjects were selected.

Data Collection and Analysis: Web of Science, PubMed, science Direct, Embase, Google scholar, MEDLINE and Cochrane library were searched for relevant records.

Main Results: A total of 2,483 records were identified through electronic database searching and 16 studies involving 81,185 reproductive age females were included. Using a random effect modelthe overall estimated pooled prevalence of maternal malnutrition (specifically undernutrition) in Africa was $20.2 \%$ (95\% CI: 14.1 27.9; $I^{2}=99.482 \%$ ).

Conclusion: Most women in Africa are suffering of malnutrition. Therefore, more focus should be directed toward ensuring appropriate and extensive application of plans for malnutrition prevention and management.

\section{Abbreviations}

ANC: Antenatal Care, BMI: Body Mass Index, CMA: Comprehensive Meta-Analysis, LBW: Low-birth-weight, MM: Maternal Malnutrition, MUAC: Mid Upper Arm Circumference, NOS: Newcastle-Ottawa Scale, PPH: Postpartum Hemorrhage, WHO: World Health Organization

Keywords: Prevalence, Determinants, Maternal Malnutrition and Africa (2000-2019).

\section{INTRODUCTION}

Globally, approximately two billion people are plagued by various forms of malnutrition which accounts for $11 \%$ of the worldwide burden of the disease [1]. Maternal malnutrition (MM) is known to make bigger the risk of poor pregnancy outcomes such as obstructed labor, premature birth or low-birth-weight (LBW) babies and postpartum hemorrhage [2]. Malnutrition amongst women of reproductive age is a primary public health difficulty in many South Asian and sub-Saharan African countries, 
where more than $20 \%$ of women are considered undernourished (Body Mass Index or BMI < $18.5 \mathrm{~kg} / \mathrm{m}^{2}$ )[3]. Maternal malnutrition for this review indicates under nutrition, therefore both term are used interchangeably. Under nutrition is the type of malnutrition normally affecting people in many low and middle income countries, in spite of the enormous development in health offerings [4, 5].Maternal under nutrition is particularly established in resourcepoor settings, typically ranging from $10 \%$ to $19 \%$ in these settings, however achieving up to greater than $20 \%$ in some areas, such as in subSaharan Africa, south-central and southeastern Asia, and Yemen[6]. Maternal malnutrition in current years has turn out to be a integral theme of Public challenge as it is associated with upward push in maternal-child morbidity and mortality. The World Health Organization (WHO) classifies malnutrition as the greatest threat to public health[7].Notwithstanding, precise dietary repute of reproductive age women is an essential thing for better effect for mothers and their children's health as well as for their family[1].A child's development depends absolutely on the mother's dietary fame throughout fetal existence and the first six months of life after delivery [8].Maternal malnutrition is now a vital difficulty in many countries In South Asia and sub-Saharan Africa and it have an effect on up to $40 \%$ of reproductive age females in some of these regions[3]. Children and women of reproductive age are the most inclined agencies to malnutrition due to vitamin $\mathrm{A}$, iodine, anemia and iron deficiencies [9].Multi-countries studies have furnished empirical evidences that dietary variety scores are dependable measures for micro- and macro-nutrient adequacy for women of reproductive ages[10]. Therefore, numerous diets are anticipated to enhance nutritional profile and as a result limit the risk of maternalchild morbidity and mortality. To address maternal malnutrition in low resources limited areas, many programs with proof of effectiveness have been recognized, such as iron folate and micro-vitamins supplementations programs and deworming program [11]. Regardless of the efforts taken in sinking malnutrition prevalence, the magnitude of malnutrition (body mass index [BMI] $<18.5$ $\mathrm{kg} / \mathrm{m} 2)$ remains $\operatorname{high}(10 \%$ to $40 \%)$ among reproductive age women in most low- and middle-income countries of Sub-Saharan Africa and South Central and South Eastern Asia[1].
Maternal under nutrition is an international health issue affecting more ladies in developing countries[12]. The burden of malnutrition in the form of under nutrition among reproductive age women is a global public health problem that need more attention[13]. Maternal under nutrition affect fetal growth and development during the first 2 years of life and is a major contributor of both stunting of linear growth and subsequent obesity and non-communicable diseases later on in adulthood[11]. Maternal under nutrition affect fetal growth and development at some stage in the first two years of life and is an essential contributor of both stunting of linear growth. Maternal under nutrition decreases efficiency, prolong restoration time from sickness and amplify susceptibility to infections and death[14].In general, malnutrition have a lengthy records of unsafe results on women and children health, therefore, working on its prevention is a durable asset that will benefits the current and future generations[15, 16]. Maternal under-nutrition is a worldwide public health problem that is persistent and destructive in many low and middle-income countries, affecting higher proportion of reproductive age females in these settings, where approximately more than 3.5 million females of reproductive age die annually due to direct or indirect causes[17].

The prevalence of maternal malnutrition in Africa is still not too clear as there exist relative difference in reported prevalence rate among published studies. Few studies have assessed the prevalence of maternal malnutrition, but the results are controversial across studies and regions. According to the 2018 World Health Organization (WHO) African regional data, between 2000 and 2015, nine countries in Africa had maternal malnutrition Prevalence rates above $15 \%$. Maternal underweight exceeds $20 \%$ in Ethiopia, Madagascar and Senegal while the lowest rates of underweight amongst women have been determined in Benin, Cameroon, Ghana, Lesotho, Rwanda and Togo[18]. In a study conducted among rural women of reproductive age in Bangladesh, the prevalence of malnutrition among the study population was estimated to be $34 \%[19]$.

Screening equipment for measuring maternal malnutrition varies from one place to another in Africa. Most studies used the body mass index (BMI) and mid-upper arm circumference (MUAC) as screening equipment for measuring maternal malnutrition in Africa. These tools are necessary in identification of not only 
malnourished females but also of females at threat of malnutrition. Women whose BMI is $<18.5 \mathrm{~kg} / \mathrm{m} 2$ or with Mid Upper Arm Circumference (MUAC) under $21 \mathrm{~cm}$ might have unhealthy pregnancy outcomes due to the risk of malnutrition[20]. Preexisting conditions like under nutrition and anemia are among the most frequent cause of $28 \%$ maternal death[20]. These maternal health issues can be minimized and prevented through iron folic supplementation programs for reproductive age women to improve pregnancy outcomes. Provision of quality antenatal care (ANC) services with splendid records keeping is also essential for good pregnancy outcome.

The World Health Assembly and the Sustainable Development Goal files definitely highlight the significance of introducing vitamin policy applications that offers exceptional interest to mother and infant diet among member nations. Implementation of such strategies place in national and global documents need to be decentralize in every setting in low and middle incomes countries, especially in rural African communities for the prevention and control of malnutrition. Concrete Information on maternal malnutrition in Africa is essential for prioritizing, designing and initiating intervention programs aimed at preventing maternal-child morbidity and mortality. We therefore, performed this systematic review with the aimed to provide evidence base report of the prevalence and determinants of maternal malnutrition in Africa that will serve as a tangible data for future research and for policy making and implementation of strategy to reduce maternalchild morbidity and mortality.

\section{MATERIALS AND MeThOdS}

A priori inclusion and exclusion criteria were developed by reviewers to pre-define the objectives and methods for the review. An initial limited systematic search was performed in a selection of relevant databases to find published research-based articles reporting on maternal malnutrition. The search was performed to primarily identify Cross-Sectional studies published from January $1^{\text {st }}, 2000$ to December $31^{\text {st }}, 2019$ that reported on outcome of interest to the review. Seven relevant electronic databases were searched to identify eligible articles for inclusion into the review. The reference lists of all identified articles were also searched for additional records that may have not been captured during the initial electronic database search. The systematic search performed yielded a total of 2,483 records, (google scholar: 2,200results, Elsevier/Science Direct: 185results, Web of science: 65results, PubMed: 16results, Embase: 5results, Cochrane library: 5results, Medline: 4results and 3 additional records obtained from reference searching). Reference management software, End Note X9 was used for recording and organizing all relevant bibliographic citations for the review. Initially, keywords used to search each data base were: Prevalence, determinants, maternal malnutrition and Africa. See Table 1 for detail of the final search terms and search strategy used per database.

Table 1. Search terms and Search strategy per database

\begin{tabular}{|l|l|}
\hline \multicolumn{1}{|c|}{ Search terms } & \multicolumn{1}{|c|}{ Search Strategy Per database } \\
\hline Prevalence & $\begin{array}{l}\text { Web of science core collection: advance search: \#1 TS=prevalence } \\
\text { (Indexes=SCI-EXPANDED, SSCI, A\&HCI, CPCI-S, CPCI-SSH, ESCI, CCR- }\end{array}$ \\
EXPANDED, IC Timespan=2000-2019), \#2 TS=maternal malnutrition \\
(Indexes=SCI-EXPANDED, SSCI, A\&HCI, CPCI-S, CPCI-SSH, ESCI, CCR- \\
EXPANDED, IC Timespan=2000-2019), \#3 TS=Africa (Indexes=SCI- \\
EXPANDED, SSCI, A\&HCI, CPCI-S, CPCI-SSH, ESCI, CCR-EXPANDED, IC \\
Timespan=2000-2019). \#3 AND \#2 AND \#1 Combine= (Indexes=SCI- \\
EXPANDED, SSCI, A\&HCI, CPCI-S, CPCI-SSH, ESCI, CCR-EXPANDED, IC \\
Timespan=2000-2019). \\
MEDLINE: advance search: \#1 TS=prevalence (Indexes=MEDLINE \\
Timespan=2000-2019), \#2 TS="maternal malnutrition" (Indexes=MEDLINE \\
Timespan=2000-2019), \#3 TS=Africa (Indexes=MEDLINE Timespan=2000- \\
2019), \#3 AND \#2 AND \#1 Combine= (Indexes=MEDLINE Timespan=2000- \\
2019). \\
Cochrane library: advance search: Title abstract keywords=Prevalence AND \\
"maternal malnutrition" AND Africa, send to search manager. \\
EMBASE: advance search: 'prevalence' AND 'maternal malnutrition' AND \\
'Africa' AND [2000-2019]/py. \\
PubMed: advance search: ((prevalence) AND "maternal malnutrition") AND \\
Africa.
\end{tabular}




\begin{tabular}{|l|l|}
\hline & Elsevier/Science Direct: advance search: Prevalence AND "maternal \\
malnutrition" AND Africa (2000-2019). \\
Google scholar advance search: "Prevalence" AND "Maternal malnutrition" \\
AND "Africa".
\end{tabular}

Institutional Review Board or Ethics Committee approval was not needed/obtained because this review data was collected from secondary sources and has to direct effect on human health.Inform consent or ethical approval was not necessary because information in this review is based on data collected from secondary sources and because this review post no direct harm to human health.

Two authors (PLB and JYL) systematically screened records to identified articles relevant for inclusion. Inclusion criteria: Cross-sectional studies Published from January $1^{\text {st }}, 2000$ to December $31^{\text {st }} 2019$ that assessed the prevalence or determinants of maternal malnutrition in Africa, where body mass index (BMI) or Mid Upper Arm Circumference (MUAC) was used to predict nutritional status of study subjects were included Exclusion criteria: Case reports, reviews and animal studies were excluded. Studies reported as abstracts were not included if there was insufficient information to allow assessment of the risk of bias.

Table 2. Assessment of methodological quality using the Newcastle - Ottawa Scale (NOS)

\begin{tabular}{|c|c|c|c|c|c|c|c|c|}
\hline \multirow[b]{2}{*}{ Study } & \multicolumn{3}{|c|}{ Selection } & \multicolumn{4}{|c|}{ Comparability } & \multirow{2}{*}{$\begin{array}{c}\begin{array}{c}\text { Outcom } \\
\text { es }\end{array} \\
\text { QAS }\end{array}$} \\
\hline & $\begin{array}{l}\text { Represe } \\
\mathrm{n} \text { - } \\
\text { tativene } \\
\text { ss Of } \\
\text { the } \\
\text { sample }\end{array}$ & $\begin{array}{l}\text { Sampl } \\
\text { e Size } \\
\text { justific } \\
\text { a-tion }\end{array}$ & $\begin{array}{l}\text { Non- } \\
\text { responde } \\
\text { nts }\end{array}$ & $\begin{array}{l}\text { Ascertain } \\
\text { ment of } \\
\text { the } \\
\text { exposure }\end{array}$ & $\begin{array}{l}\text { Comparabil } \\
\text { ity of } \\
\text { Subjects \& } \\
\text { Control of } \\
\text { Confoundi } \\
\text { ng factors }\end{array}$ & $\begin{array}{l}\text { Assessme } \\
\text { nt of the } \\
\text { outcome }\end{array}$ & $\begin{array}{l}\text { Statist } \\
\text { ical } \\
\text { test }\end{array}$ & \\
\hline $\begin{array}{l}\text { Dadi AF, et } \\
\text { al. } 2019\end{array}$ & $*$ & $*$ & $*$ & $* *$ & $*$ & $* *$ & $*$ & 9 stars \\
\hline $\begin{array}{l}\text { Hadiya H. et } \\
\text { al. } 2019\end{array}$ & $*$ & $*$ & $*$ & $* *$ & $*$ & $* *$ & $*$ & 9 stars \\
\hline $\begin{array}{l}\text { Shiferaw A, et } \\
\text { al. } 2019\end{array}$ & $*$ & $*$ & $*$ & $* *$ & $*$ & $* *$ & $*$ & 9 stars \\
\hline $\begin{array}{l}\text { H. Kedir et al. } \\
2016\end{array}$ & $*$ & $*$ & $*$ & $* *$ & $*$ & $* *$ & $*$ & 9 stars \\
\hline $\begin{array}{l}\text { Amugsi DA, } \\
\text { et al. } 2019\end{array}$ & $*$ & $*$ & & $* *$ & $*$ & $* *$ & $*$ & 8 stars \\
\hline $\begin{array}{l}\text { Petry N, et al. } \\
2019\end{array}$ & $*$ & $*$ & & $* *$ & $*$ & $* *$ & $*$ & 8 stars \\
\hline $\begin{array}{l}\text { Gebre et al. } \\
2018\end{array}$ & $*$ & $*$ & $*$ & $* *$ & $*$ & $*$ & $*$ & 8 stars \\
\hline $\begin{array}{l}\text { Ravaoarisoa } \\
\text { et al. } 2018\end{array}$ & $*$ & $*$ & & $* *$ & $*$ & $* *$ & $*$ & 8 stars \\
\hline $\begin{array}{l}\text { Ferede A, et } \\
\text { al. } 2017\end{array}$ & $*$ & $*$ & $*$ & $* *$ & $*$ & $*$ & $*$ & 8 stars \\
\hline $\begin{array}{l}\text { Saaka M, et } \\
\text { al. } 2017\end{array}$ & $*$ & $*$ & $*$ & $* *$ & $*$ & $* *$ & & 8 stars \\
\hline $\begin{array}{l}\text { Negash C, et } \\
\text { al. } 2015\end{array}$ & $*$ & $*$ & $*$ & $* *$ & $*$ & $*$ & $*$ & 8 stars \\
\hline $\begin{array}{l}\text { Senbanjo et } \\
\text { al. } 2013\end{array}$ & $*$ & $*$ & & $* *$ & $*$ & $* *$ & $*$ & 8 stars \\
\hline $\begin{array}{l}\text { S. A. Motadi } \\
\text { et al. } 2019\end{array}$ & $*$ & $*$ & & $* *$ & $*$ & $* *$ & & 7 stars \\
\hline $\begin{array}{l}\text { Kumera et al. } \\
2018\end{array}$ & $*$ & $*$ & & $* *$ & $*$ & $*$ & $*$ & 7 stars \\
\hline $\begin{array}{l}\text { Mariyam, et } \\
\text { al. } 2018\end{array}$ & $*$ & $*$ & $*$ & $* *$ & $*$ & & $*$ & 7 stars \\
\hline $\begin{array}{l}\text { AH Mtumwa } \\
\text { et al. } 2016\end{array}$ & $*$ & $*$ & & $* *$ & $*$ & $* *$ & & 7 stars \\
\hline
\end{tabular}

$Q A S=$ Quality Assessment Score, 
Two authors (PLB \& ACK) independently extracted data from each of the included study. The results were compared to minimizing the likelihood that errors would go unnoticed. In case of any discrepancy, a third author (JYL) was consulted and the issue was discussed and resolved by general consensus. Information extracted were: first author name \& year of publication, study design, sample-sized, country/location, time of study, method of data collection, prevalence of maternal malnutrition, tools used to assess malnutrition and cut-off point of tools used to assessed malnutrition.

The Newcastle-Ottawa Scale (NOS)[21], was used for assessing the quality of the included studies. The NOS included 3 categories with a maximum score of 10 stars. A grade of one to ten (1-10) stars was used to assess the methodological quality of the included studies (See Table 2). The quality of each study was rated using the following scoring algorithms: 8 to 10 stars were deemed as having good methodological quality, 5 to 7 stars were deemed as having fair methodological quality and 1 to 4 stars were deemed as having poor methodological quality. In order to advance the legitimacy of this systematic review result, we only included studies with good and fair methodological quality.

Using Comprehensive meta-analysis software version $2.0 \& 3.0$, data were collected, analyzed, and synthesized using figure and tables to facilitate classification and organization. Prevalence of maternal malnutrition obtained from each of the included studies were pooled to determine a single estimate. Heterogeneity between studies was detected using Higgins test where $\mathrm{I}^{2}$ statistic was calculated and reported. Under representation of countries by sample size used might have affected the overall result of the review leading to high heterogeneity. Pooled estimate from random effect model was reported and subgroup-analysis was done using the study setting (country in which the included studies were conducted) along with measurement tools (BMI \& MUAC) for malnutrition among reproductive age women. Using a fixed effect model, a sub-group analysis was performed to identify statistically significant determinants of maternal malnutrition.

\section{RESUltS}

A total of 2,483 records were identified through electronic database searching and a total of 2,467 studies were omitted through systematic process. From the total omitted studies, 126 were duplicates, 2185 were excluded based on title and abstract screening, 156 studies were excluded from systematic review (4 reviews, 9 animals studies, 11 case report studies, 33 studies with different study design, 22 studies not conducted in Africa and 77 studies did not report on any outcomes of interest to the current review). Finally 16 studies involving 81,185 reproductive age females were included in this systematic review (see figure 1). The qualities of the 16 included studies were assessed using the New Castle-Ottawa Scale for cross sectional studies (see Table 2). Twelve of the included studies had good methodological quality and four had fair methodological quality. The study with the smallest sample size by Negash C, et al. 2015 included 197 subjects[22], while a multicountry study by Amugsi DA, et al. 2019 had the largest sample size of 64,698 subjects[11].The smallest prevalence of maternal malnutrition was estimated by Senbanjo et al. $2013(6.7 \%)$ [23], while the largest prevalence was estimated by Ferede A, et al. 2017 (48.6\%) [1].The 16 included studies were conducted in ten countries in different regions of Africa. Regions and countries where the studies were conducted are: East Africa (Ethiopia, Kenya, Madagascar \& Tanzania), West Africa (Gambia, Ghana, \& Nigeria), Central Africa (Democratic Republic of Congo), southern region of Africa (South Africa) and Mozambique in the South-Eastern region of Africa. All the sixteen included studies used cross-sectional design. Regarding measurement of nutritional status, Mid-Upper Arm Circumference (MUAC) and Body Mass Index (BMI) were used to estimate the nutritional status of reproductive age women in all the included studies. Two studies mention the used both MUAC and BMI, while each of the others studies used either BMI or MUAC. According to NOS standard grading score for the included studies, twelve studies had strong quality and four studies had moderately strong quality (see Table 2). Using a random effect model with the help of the comprehensive meta-analysis (CMA $2 \& 3$ ) software, the overall pooled prevalence of maternal malnutrition (particularly under nutrition) among reproductive age women in Africa was estimated to be $20.2 \%$ ( 95\%CI: 14.1- 27.9; $\left.\mathrm{I}^{2}=99.482 \%\right)$ see table 4 . Based on the current systematic review pooled effect size findings; Poor habit of hand washing practices 0.706 (95\% CI: $0.657,0.751$ ), food insecurity 0.676 (95\%CI: $0.632,0.718)$, low family income 0.655 (95\%CI: $0.580,0.723$ ), Illiterate 
husband0.392(95\%CI: $0.357, \quad 0.427$ ), low decision making autonomy0.345(95\%CI: 0.314 , 0.377), multi-parity0.292(95\%CI: 0.195, 0.413), bad marital condition from a wife's perspective0.081(95\%CI: $0.046, \quad 0.141$ ),

Table 3. Characteristics of the included studies frequently coffee consumption $0.252(95 \% \mathrm{CI}$ : $0.186,0.332$ ) maternal age $0.152(95 \%$ CI: 0.108 , 0.210 ) are statistically significant factors that determine maternal under nutrition (see table 6).

\begin{tabular}{|c|c|c|c|c|c|c|c|c|c|}
\hline & $\begin{array}{l}\text { Study } \\
\text { Design }\end{array}$ & Cases & $\begin{array}{l}\text { Sample } \\
\text { Size }\end{array}$ & MDC & $\begin{array}{l}\text { Measuring } \\
\text { tools }\end{array}$ & $\begin{array}{l}\text { Cut-off } \\
\text { value }\end{array}$ & Prevalence & $\begin{array}{l}\text { Country/ } \\
\text { Location }\end{array}$ & QAS \\
\hline $\begin{array}{l}\text { Dadi AF, et al. } \\
2019\end{array}$ & $\begin{array}{l}\text { Cross- } \\
\text { Sectional }\end{array}$ & 135 & 940 & IAQ & MUAC & $<22$ & $14.40 \%$ & Ethiopia & Good \\
\hline $\begin{array}{l}\text { Ferede A, et al. } \\
2017\end{array}$ & $\begin{array}{l}\text { Cross- } \\
\text { Sectional }\end{array}$ & 209 & 430 & Q & BMI & $\begin{array}{l}<18.5 \\
\mathrm{~kg} / \mathrm{m} 2\end{array}$ & $48.60 \%$ & Ethiopia & Good \\
\hline Gebre et al. 2018 & $\begin{array}{l}\text { Cross- } \\
\text { Sectional }\end{array}$ & 216 & 900 & IAQ & MUAC & $<21 \mathrm{~cm}$ & $24.00 \%$ & Ethiopia & Good \\
\hline $\begin{array}{l}\text { H. Kedir et al. } \\
2016\end{array}$ & $\begin{array}{l}\text { Cross- } \\
\text { Sectional }\end{array}$ & 733 & 1,731 & & MUAC & $<22 \mathrm{~cm}$ & $42.35 \%$ & Ethiopia & Good \\
\hline $\begin{array}{l}\text { Ravaoarisoa } \\
\text { al. } 2018\end{array}$ & $\begin{array}{l}\text { Cross- } \\
\text { Sectional }\end{array}$ & 114 & 670 & & $\begin{array}{l}\text { BMI } \\
\text { MUAC }\end{array}$ & $\begin{array}{l}<18.5 \\
\mathrm{~kg} / \mathrm{m} 2 \\
<220 \mathrm{~mm}\end{array}$ & $\begin{array}{l}17.00 \% \\
9.00 \%\end{array}$ & Madagascar & Good \\
\hline $\begin{array}{l}\text { Shiferaw A, et al. } \\
2019\end{array}$ & $\begin{array}{l}\text { Cross- } \\
\text { Sectional }\end{array}$ & 168 & 374 & $\begin{array}{l}\mathrm{I}, \mathrm{O}, \& \\
\text { AMSC }\end{array}$ & MUAC & $<23 \mathrm{~cm}$ & $44.92 \%$ & Ethiopia & Good \\
\hline $\begin{array}{lll}\begin{array}{l}\text { Kumera } \\
2018\end{array} & \text { et } & \text { al. } \\
\end{array}$ & $\begin{array}{l}\text { Cross- } \\
\text { Sectional } \\
\end{array}$ & 65 & 402 & IAQ & MUAC & $<22 \mathrm{~cm}$ & $16.20 \%$ & Ethiopia & Fair \\
\hline $\begin{array}{l}\text { AH Mtumwa et } \\
\text { al.2016 }\end{array}$ & $\begin{array}{l}\text { Cross- } \\
\text { Sectional }\end{array}$ & 785 & 6,933 & $\begin{array}{l}\text { TDHS } \\
2010\end{array}$ & BMI & $\begin{array}{l}\leq 18.5 \\
\mathrm{~kg} / \mathrm{m} 2\end{array}$ & $11.32 \%$ & Tanzania & Fair \\
\hline $\begin{array}{l}\text { Saaka M, et al. } \\
2017\end{array}$ & $\begin{array}{l}\text { Cross- } \\
\text { Sectional }\end{array}$ & 115 & 400 & I \& Q & MUAC & $<25.0 \mathrm{~cm}$ & $28.80 \%$ & Ghana & Good \\
\hline $\begin{array}{l}\text { Mariyam et al. } \\
2018\end{array}$ & $\begin{array}{l}\text { Cross- } \\
\text { Sectional }\end{array}$ & 196 & 616 & $\begin{array}{ll}\text { Q } & \& \\
\text { CL } & \\
\end{array}$ & MUAC & $<21 \mathrm{~cm}$ & $31.82 \%$ & Ethiopia & Fair \\
\hline $\begin{array}{ll}\text { Senbanjo et al. } \\
2013\end{array}$ & $\begin{array}{l}\text { Cross- } \\
\text { Sectional }\end{array}$ & 20 & 300 & & BMI & $\begin{array}{l}<18.5 \\
\mathrm{~kg} / \mathrm{m} 2\end{array}$ & $6.70 \%$ & Nigeria & Good \\
\hline $\begin{array}{l}\text { Petry N, et al. } \\
2019\end{array}$ & $\begin{array}{l}\text { Cross- } \\
\text { Sectional }\end{array}$ & 263 & 1703 & $\mathrm{Q}$ & $\begin{array}{l}\text { BMI } \\
\text { MUAC }\end{array}$ & $\begin{array}{l}<18.5 \\
\mathrm{~kg} / \mathrm{m} 2 \\
<23 \mathrm{~cm}, \\
\end{array}$ & $11.50 \%$ & Gambia & Good \\
\hline $\begin{array}{l}\text { Hadiya H. et al. } \\
2019\end{array}$ & $\begin{array}{l}\text { Cross- } \\
\text { Sectional }\end{array}$ & 83 & 478 & SQ & BMI & $\begin{array}{l}<18.5 \\
\mathrm{~kg} / \mathrm{m} 2\end{array}$ & $17.40 \%$ & Ethiopia & Good \\
\hline $\begin{array}{l}\text { Amugsi DA, et } \\
\text { al. } 2019\end{array}$ & $\begin{array}{l}\text { Cross- } \\
\text { Sectional }\end{array}$ & $\begin{array}{l}5,888 \\
1,208 \\
160 \\
1,180 \\
628 \\
2,712\end{array}$ & $\begin{array}{l}64,698 \\
9,506 \\
3,012 \\
9,993 \\
11,017 \\
31,170\end{array}$ & SDQ & $\begin{array}{l}\text { BMI } \\
\text { BMI } \\
\text { BMI } \\
\text { BMI } \\
\text { BMI } \\
\text { BMI }\end{array}$ & $\begin{array}{l}<18.5 \\
\mathrm{~kg} / \mathrm{m}^{2} \\
\\
<18.5 \\
\mathrm{~kg} / \mathrm{m} 2 \\
\\
<18.5 \\
\mathrm{~kg} / \mathrm{m} 2 \\
\\
<18.5 \\
\mathrm{~kg} / \mathrm{m} 2 \\
\\
<18.5 \\
\mathrm{~kg} / \mathrm{m} 2 \\
\\
<18.5 \\
\mathrm{~kg} / \mathrm{m} 2\end{array}$ & $\begin{array}{l}9.10 \% \\
12.70 \% \\
5.31 \% \\
11.80 \% \\
5.70 \% \\
8.70 \%\end{array}$ & $\begin{array}{l}\text { DRC } \\
\text { Ghana } \\
\text { Kenya } \\
\text { Mozambique } \\
\text { Nigeria }\end{array}$ & Good \\
\hline $\begin{array}{l}\text { Negash C, et al. } \\
2015\end{array}$ & $\begin{array}{l}\text { Cross- } \\
\text { Sectional }\end{array}$ & 28 & 197 & SQ & BMI & $\begin{array}{l}<18.5 \\
\mathrm{~kg} / \mathrm{m} 2\end{array}$ & $14.20 \%$ & Ethiopia & Good \\
\hline $\begin{array}{l}\text { S. A. Motadi et } \\
\text { al. } 2019\end{array}$ & $\begin{array}{l}\text { Cross- } \\
\text { Sectional }\end{array}$ & 39 & 240 & $\begin{array}{l}\text { IAQ \& } \\
\text { FFQ }\end{array}$ & BMI & $\begin{array}{l}<18.5 \\
\mathrm{~kg} / \mathrm{m} 2 \\
>30 \mathrm{~kg} / \mathrm{m} 2\end{array}$ & $16.30 \%$ & South Africa & Fair \\
\hline
\end{tabular}

QAS=Quality Assessment Score, IAQ=interview administered questionnaire, MUAC=Mid Upper Arm circumference, BMI=Body Mass Index, I=Interview, HMR=Hospital Medical Records, $\mathrm{CL}=$ Checklist, SI=Structure Interview, SQ=Structure Questionnaire, $\mathrm{SDQ}=$ Standardized Questionnaires, FFQ=Food Frequency Questionnaire, AMSC=Anthropometric measurements and standard checklists, $\mathrm{O}=$ Observation, TDHS=Tanzania Demographic and Health Survey, DRC=Democratic Republic of Congo 
Prevalence and Determinants of Maternal Malnutrition in Africa: A Systematic Review

Table 4. Comprehensive Meta-analysis of Maternal Malnutrition Prevalence rate in Africa (Random effect model, $N=16$ ).

\begin{tabular}{|l|l|l|l|l|l|}
\hline Model & Study ID & Event rate & Lower limit & Upper limit & p-value \\
\hline & Dadi AF, et al. 2019 & 0.144 & 0.123 & 0.168 & 0.000 \\
\hline & Ferede A, et al. 2017 & 0.486 & 0.439 & 0.533 & 0.563 \\
\hline & Gebre et al. 2018 & 0.240 & 0.213 & 0.269 & 0.000 \\
\hline & H. Kedir et al. 2016 & 0.423 & 0.400 & 0.447 & 0.000 \\
\hline & Ravaoarisoa et al. 2018 & 0.170 & 0.144 & 0.201 & 0.000 \\
\hline & Shiferaw A, et al. 2019 & 0.449 & 0.399 & 0.500 & 0.050 \\
\hline & Kumera et al. 2018 & 0.162 & 0.129 & 0.201 & 0.000 \\
\hline & AH Mtumwa et al.2016 & 0.113 & 0.106 & 0.121 & 0.000 \\
\hline & Saaka M, et al. 2017 & 0.288 & 0.245 & 0.334 & 0.000 \\
\hline & Mariyam , et al. 2018 & 0.318 & 0.283 & 0.356 & 0.000 \\
\hline & Senbanjo et al. 2013 & 0.067 & 0.043 & 0.101 & 0.000 \\
\hline & Petry N, et al. 2019 & 0.154 & 0.138 & 0.172 & 0.000 \\
\hline & Hadiya H. et al. 2019 & 0.174 & 0.142 & 0.210 & 0.000 \\
\hline & Amugsi DA, et al. 2019 & 0.091 & 0.089 & 0.093 & 0.000 \\
\hline & Negash C, et al. 2015 & 0.142 & 0.100 & 0.198 & 0.000 \\
\hline & S. A. Motadi et al. 2019 & 0.163 & 0.121 & 0.215 & 0.000 \\
\hline & Overall pooled estimate & $\mathbf{0 . 2 0 2}$ & $\mathbf{0 . 1 4 1}$ & $\mathbf{0 . 2 7 9}$ & $\mathbf{0 . 0 0 0}$ \\
\hline
\end{tabular}

Table 5. A Comprehensive sub-group analysis on Prevalence of Maternal Malnutrition among reproductive age females in Africa (Random effect model, $N=16$ ).

\begin{tabular}{|l|l|l|l|l|}
\hline \multicolumn{1}{|c|}{ Variables } & \multicolumn{1}{|c|}{ \# of Studies } & \multicolumn{1}{c|}{ Sample size } & Pooled estimate, (95\% CI) & p-value \\
\hline Country where study was conducted & & & & \\
\hline Ethiopia & 9 & 6068 & $0.302(0.291,0.314)$ & 0.000 \\
\hline Other African countries & 7 & 74944 & $0.096(0.094,0.099)$ & 0.000 \\
\hline Tools used for Measuring malnutrition & & & & \\
\hline BMI & 9 & 75,649 & $0.098(0.096,0.100)$ & 0.000 \\
\hline MUAC & 7 & 5363 & $0.304(0.291,0.316)$ & 0.000 \\
\hline
\end{tabular}

BMI=Body Mass Index, MUAC= Mid-Upper Arm Circumference

\section{PRISMA 2009 Flow Diagram}

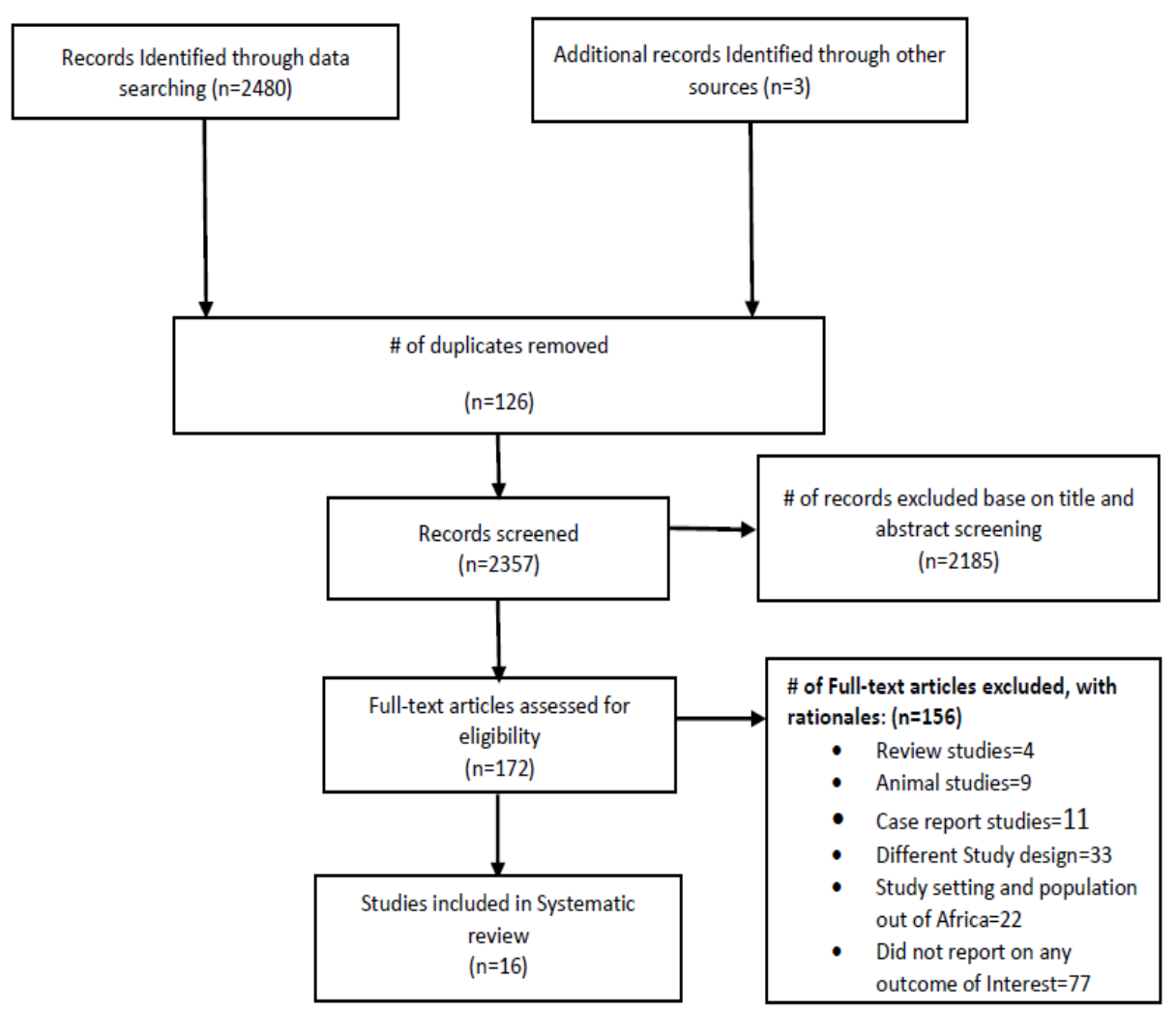

Figure 1. Process of study selection for inclusion in this systematic review 
Table 6. Sub-group analysis on determinants of maternal undernutrition among reproductive age (15-49years) females in Africa (Using the Fixed effect model).

\begin{tabular}{|l|l|l|l|l|l|}
\hline \multicolumn{1}{|c|}{ Variables } & $\begin{array}{c}\text { \# of } \\
\text { studies }\end{array}$ & $\begin{array}{c}\text { Total number of } \\
\text { under nourished } \\
\text { subjects per variable }\end{array}$ & \multicolumn{1}{|c|}{ Pooled estimate(95\% CI) } & $\mathbf{I}^{2}$ & p-value \\
\hline maternal age & 2 & 344 & $0.152(0.108,0.210)$ & 96.327 & 0.000 \\
\hline Low maternal educational level & 2 & 148 & $0.513(0.419,0.607)$ & 97.283 & 0.784 \\
\hline Illiterate husband & 1 & 733 & $0.392(0.357,0.427)$ & 0.000 & 0.000 \\
\hline Food insecurity & 3 & 460 & $0.676(0.632,0.718)$ & 76.162 & 0.000 \\
\hline Unsafe source of drinking water & 2 & 323 & $0.502(0.439,0.565)$ & 98.313 & 0.955 \\
\hline Poor habit of hand washing & 2 & 377 & $0.706(0.657,0.751)$ & 92.981 & 0.000 \\
\hline ANC visit \& service provision & 2 & 412 & $0.457(0.408,0.507)$ & 96.428 & 0.092 \\
\hline Low family income & 1 & 168 & $0.655(0.580,0.723)$ & 0.000 & 0.000 \\
\hline $\begin{array}{l}\text { Bad Marital condition from } \\
\text { wife's perspective }\end{array}$ & 1 & 135 & $0.081(0.046,0.141)$ & 0.000 & 0.000 \\
\hline Coffee drinking & 1 & 135 & $0.252(0.186,0.332)$ & 0.000 & 0.000 \\
\hline $\begin{array}{l}\text { Low Decision making } \\
\text { autonomy }\end{array}$ & 2 & 901 & $0.345(0.314,0.377)$ & 94.808 & 0.000 \\
\hline Maternal Anemia & 2 & 261 & $0.529(0.468,0.589)$ & 0.000 & 0.354 \\
\hline Rural residence & 1 & 65 & $0.508(0.388,0.626)$ & 0.000 & 0.901 \\
\hline Multi-parity & 1 & 65 & $0.292(0.195,0.413)$ & 0.000 & 0.001 \\
\hline Intestinal parasite & 1 & 65 & $0.538(0.417,0.655)$ & 0.000 & 0.536 \\
\hline
\end{tabular}

\#=number, $95 \%$ CI=95\% Confidence interval

\section{DISCUSSION}

This review assessed the prevalence of maternal malnutrition (specifically under nutrition) among reproductive age women in Africa and estimate $20.2 \%$ overallprevalence rate. This review finding is in agreement with the widespread assumption that the prevalence of maternal malnutrition appears to be much lower in almost all regions of the world except in Africa. The prevalence of maternal malnutrition in Africa was reported as $23.5 \%$ in one systematic review and meta-analysis[24] while we estimate $20.2 \%$ in this current review. Despite the drop in prevalence rate of maternal malnutrition in Africa as reported by this current review compared to past review findings, more efforts is still required to prevent and manage malnutrition because our prevalence estimate exceed $10 \%$, which is a cut-off score for declaring maternal malnutrition as a major public health problem[25, 26].

The prevalence of maternal malnutrition in Africa has not been so clear over the past few years. Several studies have tried estimating the prevalence of maternal malnutrition but their findings varies relatively from one country to another. A study conducted on the prevalence and determinants of malnutrition among reproductive aged women of rural Bangladesh estimated $34.0 \%$ prevalence rate among the study subjects[27]. Several studies conducted in Africa on maternal malnutrition, estimated different prevalence rates for malnutrition among reproductive age women such as: $6.70 \%$
[23] ,16.2\% [28], 16.30\%[29], 42.35\%[30], and 44.92\%[17]. This variation in prevalence rate among studies is most likely associated with the screening tools used to measured malnutrition. This can be witnessed by the result from our sub-group analysis (see table5).

Maternal age showed a statistically significant association with maternal under nutrition among mothers. Based on these review findings, it is evidence that the age of a mother significantly influence hernutritional status. As the age of a mother increased by one year, her risk of under nutrition decreases. In the case of young mothers, apart from inadequate development of their reproductive system and their need of nutrition for their growing body, they are often bizarrely encircled by uncomplimentary nutritional conditions [31-33].This finding is consistent with study conducted in Bangladesh [34], southern Ethiopia [35] and Lebanon [32].Bad marital condition from a wife perspective was found to be an important predictor of mothers' nutritional status. A bad marital condition explained as unhappiness in a marital relationship would affect the support or care that the mother should get from her partner and this can indirectly affect her nutritional condition. Psychologically it might decrease the woman eating hobbits thus increasing her risk of malnutrition. We found that frequent coffee drinking increased the risk of under nutrition among pregnant mothers. Mothers who occasionally drink coffee are less likely to develop malnutrition compared to those who 
consumed coffee frequently. This is scientifically evidenced that moderate caffeine intake $(300 \mathrm{mg} /$ day) or taking about three cups of coffee is probably not harmful to pregnant mothers [36, 37] but taking a large amounts of caffeine may cause nutrient depletion and interfere with nutrient absorption [36, 38] thus increasing the risk of malnutrition. Household food insecurity was also another variable that showed statistically significant association with maternal under nutrition. Women from household with food insecurity have $67.6 \%$ increased risk of becoming undernourished as compared to their counterparts. This finding is consistent with findings of studies conducted in Tigray region [39], Gambella [40] and in Nepal (Acharya SR BJ, Timilsina DP. 2017).Women who had low decision making autonomy at household level have $34.5 \%$ increased risk of being malnourished as compared to those who had high decision making autonomy. This finding is consistent with findings from studies conducted ineastern Ethiopia by Kedir H BY in 2016 and in rural India by Oakley L BC in 2017.This review found that the probability of being undernourished among women who demonstrated poor habit of hand washing practices is $70.6 \%$ higher than that of their counter parts in Africa. This finding on hand washing habit should be highlighted for preventive intervention measures in all of Africa. This review found that women from household with low family income $>30.00$ USD or $>1000$ Ethiopian Birr monthly have $65.5 \%$ increase risk of being undernourished as compared to those from household with better family income. This finding is consistent with the findings from studies conducted in eastern Ethiopia by Kedir H BY in 2016 and in rural Bangladesh [27].Another finding of this review that affects women's nutritional status is multiparity. The risk of under nutrition increases as parity advances. Multiparous women have $29.2 \%$ increase risk of being malnourished as compared to their counterparts. This finding is consistent with the facts that repeated reproductive cycles deplete maternal nutrition store. Moreover, higher-level of parity compels women to take care of their children rather than protecting their own health and nutritional status mostly in limited household resources event. This finding is also found in previous studies by Belete Y NB and Firehiwot M in 2016 and by Okwu GN UA in 2008. The current review report that women with illiterate husbands has $39.2 \%$ risk of being malnourished. Women, whose husbands are educated, may have better access to information regarding nutrition during pregnancy through their husbands. Therefore, women in this category may get better health and nutritional support than their counterparts.

Nutrition has profound effects on human's health throughout the course of life and it is indistinguishably associated with cognitive and social development of children, specifically in early childhood. Nutritional standing of reproductive age women before conception and during pregnancy is critical for fetal growth and development. Mtumwa AH in 2016 reported that malnutrition among women don't only have a major impact on their health, but also on their children's health, because a chronically undernourished woman is most likely to give birth to an undernourished child, causing the cycle of under nutrition to be repeated over generations. In most low resource settings, maternal malnutrition is associated with an increased risk of maternal morbidity and mortality and with poor pregnancy outcomes such as low birth weight and preterm birth babies[25]. Children born in such condition might not be able to achieve their full growth and developmental potential because of effects of maternal malnutrition during fetal life. Suboptimal dietary practices, lack of education and limited access to nutritional diet and information, compromise the nutritional status of reproductive age women in resource poor settings (Hadiya Hassen Tikuye 2019). In agreement with these findings, it would be correct to estimate that $20.2 \%$ of reproductive age females in Africa are suffering from malnutrition (specifically under nutrition).

\section{LIMITATIONS}

This review is subject to few limitations. Firstly, there was no eligible study from some regions in Africa to be included in this review. For example, none of the included studies in this review came from the Northern region of Africa. For some African countries, there were only few studies with sample size which might not have been representative of the whole population in that region. Additionally, few of the included studies had large sample size which might have influenced the overall result of this review.

\section{Conclusions}

The interrelationship between a mother and her child nutritional status stresses the value of improving maternal nutritional status as this could improve both maternal and child health 
outcomes. Adequately addressing challenges of maternal malnutrition in Africa is essential to ensuring optimal cognitive growth and development for women of reproductive age. Therefore, more is required of policy makers, health care providers, civil society organizations and programs implementers in combating this deadly disease.

\section{AUThORS' CONTRIBUTIONS}

PLB developed the search strategy, which was reviewed by JYL. PLB and ACK extracted data and did the statistical analysis. PLB \& JYL assessed the quality of the included studies. PLB drafted the final manuscript and after reviewing it, all the authors agree it was ready for submission.

\section{ACKNOWLEDGEMENTS}

The authors would like to express their gratitude to the Central South University family for their support. Finally, thanks to the corresponding author (Professor Jiayou Luo) for his tireless effort and assistance during the period of this review. This systematic review is sponsor by funding from the National Natural Science Foundation of China (grants number 81172680).

\section{REFERENCES}

[1] Ferede A, Lemessa F, Tafa M, Sisay S: The prevalence of malnutrition and its associated risk factors among women of reproductive age in Ziway Dugda district, Arsi Zone, Oromia Regional State, Ethiopia. Public Health 2017, 152:1-8.

[2] Gebre B, Biadgilign S, Taddese Z, Legesse T, Letebo M: Determinants of malnutrition among pregnant and lactating women under humanitarian setting in Ethiopia. BMC Nutrition 2018, 4(1).

[3] Ravaoarisoa L, Randriamanantsaina L, Rakotonirina J, Rakotomanga JdDM, Donnen P, Dramaix MW: Socioeconomic determinants of malnutrition among mothers in the Amoron'i Mania region of Madagascar: a cross-sectional study. BMC Nutrition 2018, 4(1).

[4] FAO I U, WFP and WHO. 2018.: The State of Food Security and Nutrition in the World 2018. Building climate resilience for food security and nutrition. Rome, FAO. . FAO 20182018.

[5] Branca F G-SL, Borghi E, Blossner Md, Onis Md. : Extension of the WHO maternal, infant and young child nutrition targets to 2030 . SCN News. 2015;(41):55-8. 2015.

[6] Alice M. Tang MC, Kimberly Dong, Norma Terrin, Andrew Edmonds, Nega Assefa, Terusha Chetty, Roshan Ramlal, Parul Christian, Keith West, Naveed Janjua, Christine
Wanke, Megan Deitchler, Zeina MaaloufManasseh: <Determining a Global Mid-Upper Arm Circumference Cutoff to Assess Malnutrition in Pregnant Women.pdf>. journal of Nutrition [Food And Nutrition Technical Assistance, (FANTA)] 2016.

[7] Dadi AF, Desyibelew HD: Undernutrition and its associated factors among pregnant mothers in Gondar town, Northwest Ethiopia. PLoS One 2019, 14(4):e0215305.

[8] Mason JB, Shrimpton R, Saldanha LS, Ramakrishnan U, Victora CG, Girard AW, McFarland DA, Martorell R: The first 500 days of life: policies to support maternal nutrition. Glob Health Action 2014, 7:23623.

[9] Petry N, Jallow B, Sawo Y, Darboe MK, Barrow S, Sarr A, Ceesay PO, Fofana MN, Prentice AM, Wegmuller R et al: Micronutrient Deficiencies, Nutritional Status and the Determinants of Anemia in Children 0-59 Months of Age and Non-Pregnant Women of Reproductive Age in The Gambia. Nutrients 2019, 11(10).

[10] Saaka M, Oladele J, Larbi A, Hoeschle-Zeledon I: Dietary Diversity Is Not Associated with Haematological Status of Pregnant Women Resident in Rural Areas of Northern Ghana. Journal of Nutrition and Metabolism 2017, 2017:1-10.

[11] Black RE, Victora CG, Walker SP, Bhutta ZA, Christian P, de Onis M, Ezzati M, GranthamMcGregor S, Katz J, Martorell $\mathrm{R}$ et al: Maternal and child undernutrition and overweight in low-income and middle-income countries. Lancet 2013, 382(9890):427-451.

[12] Adebowale S.A. AOT, Okareh O.T. \& Fagbamigbe F.A. : Social epidemiology of adverse nutritional status outcomes among women in Nigeria: NDHS, 2008.Pakistan Journal of Nutrition 2011.

[13] Amugsi DA, Dimbuene ZT, Kyobutungi C: Correlates of the double burden of malnutrition among women: an analysis of cross sectional survey data from sub-Saharan Africa. BMJ Open 2019, 9(7):e029545.

[14] Mozayeni M MA, Eshraghian MR, Davaei M. : Relationship between food security and stress in pregnant mothers and low birth weight infant in childbirth conducted in 2010 in Tehran Akbar Abadi hospital.Int J Curr Res 2014.

[15] Black RE LC, Walker N, Chou D, Liu L, Temmerman M. : Reproductive, maternal, newborn, and child health: key messages from Disease Control Priorities. . Lancet 2016.

[16] C. F: Maternal nutrition: Effects on health in the next generation.Indian J Med Res 2009.

[17] Addisu Shiferaw1 GH: <Acute Under Nutrition and Associated Factors among Pregnant Women in Gumay District, Jimma Zone, South 
West Ethiopia.pdf>. Journal of Women's Health Care 2019.

[18] < Nutrition in the WHO African Regional Office for Africa Brazzaville 2017.pdf>. World Health Organization(WHO) Rehional Office for Africa 2017.

[19] Abul Hasnat Milton M, PhD, Wayne Smith, BMed, PhD, Bayzidur Rahman, BSc, Bashir Ahmed, MSc, PhD, S. M. Shahidullah, MSc, Zakir Hossain, BSc, Ziaul Hasan, MBBS, MPH, and Sabrina Sharmin, MBBS: $<$ Prevalence and Determinants of Malnutrition Among Reproductive Aged Women of Rural Bangladesh.pdf $>$. Asia-Pacific Journal of Public Health 2010.

[20] Mariyam AF, Dibaba B: Epidemiology of Malnutrition among Pregnant Women and Associated Factors in Central Refit Valley of Ethiopia, 2016. Journal of Nutritional Disorders \& Therapy 2018, 08(01).

[21] <NEWCASTLE - OTTAWA QUALITY ASSESSMENT SCALE adopted for Crosssectional studies.PDF>.

[22] Negash C, Whiting SJ, Henry CJ, Belachew T, Hailemariam TG: Association between Maternal and Child Nutritional Status in Hula, Rural Southern Ethiopia: A Cross Sectional Study. PLoS One 2015, 10(11):e0142301.

[23] Idowu O Senbanjo1, Ibiyemi O Olayiwola2, Wasiu A Afolabi2 and Olayinka C Senbanjo3: $<$ Maternal and child under-nutrition in rural and urban comunities of Lagos State, Nigeria the relationship and risk factors.pdf $>$. BMC Research Notes 2013.

[24] Desyibelew HD, Dadi AF: Burden and determinants of malnutrition among pregnant women in Africa: A systematic review and meta-analysis. PLoS One 2019, 14(9):e0221712.

[25] Razak F FJ, Subramanian S.: Maternal underweight and child growth and development. . The Lancet 2013.

[26] FAO I U, WFP and WHO.: The State of Food Security and Nutrition in the World 2018. Building climate resilience for food security and nutrition. Rome, FAO. 2018.

[27] Abul Hasnat Milton M, PhD, Wayne Smith, BMed, PhD, Bayzidur Rahman, BSc, Bashir Ahmed, MSc, PhD, S. M. Shahidullah, MSc, Zakir Hossain, BSc, Ziaul Hasan, MBBS, MPH, and Sabrina Sharmin, MBBS: $<$ Prevalence and Determinants of malnutrition among reproductive age women of rural Bandladesh.pdf $>$. Asia-Pacific Journal of Public Health 2010.

[28] Kumera G, Gedle D, Alebel A, Feyera F, Eshetie S: Under nutrition and its association with socio-demographic, anemia and intestinal parasitic infection among pregnant women attending antenatal care at the University of
Gondar Hospital, Northwest Ethiopia. Matern Health Neonatol Perinatol 2018, 4:18.

[29] Selekane Ananias Motadi ZM, Palesa Harriet Mogane, Phumuzo Masidwali, Masindi Makwarela, and Lindelani Mushaphi: Assessment of Nutritional Status and Dietary Intake of Pregnant Women in Rural Area of Vhembe District, Limpopo Province. Ecology of Food and Nutrition: Routledge Taylor \& Francis Group 2019.

[30] Haji Kedir* YBaAW: <Magnitude and determinants of malnutrition among pregnant women in eastern Ethiopia evidence from rural, community-based setting.pdf>. (C) 2014 John Wiley \& Sons Ltd Maternal and Child Nutrition 2016.

[31] Symington EA BJ, Malan L, Zandberg L, Ricci C, Smuts CM. PMID:, 30041623: Nutrition during pregnancy and early development (NuPED) in urban South Africa: a study protocol for a prospective cohort. BMC pregnancy and childbirth https://doiorg/101186/ s12884-018-1943-62018.

[32] Zgheib C MJ, Sacre Y. : Evaluation of Food Behaviour and Nutritional Status of Pregnant Women Resident in Keserwan. . J Preg Child Health 2017.

[33] Abasizadeh S HZ, Deris F. : Prevalence of malnutrition during pregnancy and associated factors in women of Ardal County in 20122013.International Journal of Epidemiologic Research 2016.

[34] Hossain B ST, Reja S, Akter M. : Nutritional status of pregnant women in selected rural and urban area of Bangladesh. J Nutr Food Sci 2013;

[35] Regassa N SB: Contextual risk factors for maternal malnutrition in a food-insecure zone insouthern Ethiopia. Journal of biosocial science https://doiorg/101017/ S00219320120 0017X PMID: 22716940 2012;.

[36] Da Mota Santana J. Alves de Oliveira Queiroz V. MBS, Barbosa Dos Santos D., Marlucia, A. OA: Food consumption patterns during pregnancy: A longitudinal study in a region of the north east of Brazil. . Nutr Hosp https://doiorg/103305/nh20153218970 PMID: 26262702015 ;

[37] T. W: Effects of caffeine on health and nutrition: A Review. . Food Science and Quality Management 2014;

[38] Prenkert M EM: Growth data of underprivileged children living in rural areas of Chin State,Burma/Myanmar, compared to the WHO reference growth standards: an observational study. . BMJ open 2016; .

[39] Saba Abraham S MG, Shumye A. : Magnitude of chronic energy deficiency and its associated factors among women of reproductive age in 
the Kunama population, Tigray, Ethiopia, in 2014. . BMC Nutrition 2015;1:1-.

[40] Nigatu M GT, Gemeda HD. : Household food insecurity, low dietary diversity, and early marriage Were predictors for undernutrition among pregnant women residing in Gambella, Ethiopia. . Adv Public Health 2018.

Citation: Prince L. Bestman, Atipatsa C. Kaminga ,Jiayou Luo. Prevalence and Determinants of Maternal Malnutrition in Africa: A Systematic Review. ARC Journal of Gynecology and Obstetrics. 2020; 5(1):1-12. DOI:dx.doi.org/10.20431/2456-0561.0501001.

Copyright: (C) 2020 Authors. This is an open-access article distributed under the terms of the Creative Commons Attribution License, which permits unrestricted use, distribution, and reproduction in any medium, provided the original author and source are credited. 